\title{
Relationship between transpulmonary artery distanc
and pulmonary arterial pressure in patients with chronic bronchitis
}

\author{
P. H I CKEN, I. D. GREEN, A N D J M . B I S H O P \\ From the Department of Medicine, University of Birmingham, Queen Elizabeth Hospital, Birmingham
}

\begin{abstract}
Measurements were made of the distance between the bifurcation of the right and left main pulmonary arteries (the transpulmonary artery distance) on plain chest radiographs in healthy subjects, patients with chronic bronchitis, and patients with polycythaemia rubra vera. The following figures for transpulmonary artery distance were obtained-healthy subjects: range $7.0-10.5 \mathrm{~cm}$., mean $8.76 \mathrm{~cm}$., S.D. $0.81 \mathrm{~cm}$.; patients with chronic bronchitis: range $7.3-12.4$ cm., mean $10.26 \mathrm{~cm}$., S.D. $1.08 \mathrm{~cm}$.; patients with polycythaemia rubra vera: range $8.3-10.7$ cm., mean $9.20 \mathrm{~cm}$., S.D. $0.57 \mathrm{~cm}$. The transpulmonary artery distance was related to height but not to age or sex in the healthy subjects. It did not appear to be influenced by variations in the central blood volume. There was a linear relation between transpulmonary artery distance and mean pulmonary arterial pressure in patients with chronic bronchitis. In 21 of 29 patients whose transpulmonary artery distance was greater than $9.5 \mathrm{~cm}$., the mean pulmonary arterial pressure exceeded $30 \mathrm{~mm}$. $\mathrm{Hg}$. In only one of the 13 patients whose transpulmonary artery distance was less than $9.5 \mathrm{~cm}$. did the mean pulmonary arterial pressure exceed $30 \mathrm{~mm}$. $\mathrm{Hg}$. These results suggest that the measurement of transpulmonary artery distance may be useful in following the progression of pulmonary hypertension and detecting its presence in epidemiological and other studies.
\end{abstract}

Pulmonary hypertension is a frequent but by no means invariable feature of patients with chronic bronchitis. While it occurs relatively early and progresses over a period of a few years in some patients, in others equally disabled the pulmonary arterial pressure may remain almost normal. A method which would enable an estimate to be made of pulmonary arterial pressure without resort to cardiac catheterization would have considerable value in following the progress of individual patients in epidemiological studies and in investigations designed to identify subgroups from the generality of patients with chronic bronchitis. The electrocardiogram might provide evidence of the presence of right ventricular hypertrophy, but its limitations in this kind of patient have recently been demonstrated (Millard, 1967).

Milne (1963) showed that the size of the pulmonary artery on chest radiographs was related to the mean pulmonary arterial pressure in patients with mitral stenosis. The present investigation was designed to see if there was any relation between a measurement of size of the main pulmonary arteries on plain chest radiographs and the mean pulmonary arterial pressure in patients with chronic bronchitis.

\section{MATERIAL AND METHODS}

Observations were made in three groups of patients․ㅡㄹ Many of these patients had undergone cardias catheterization during recent years in the course of studies which have been published previouslo (Bishop, Harris, and Segel, 1965 ; Segel and Bishop? 1965, 1966 ; Abraham, Hedworth-Whitty, and BishopN 1967; and Segel and Bishop, 1967) or are still in progress. No patient had a cardiac catheterization solely for the purpose of the present investigation. N

GROUP A This consisted of 42 healthy persons (2 men and 21 women) aged from 15 to 62 years Twenty-one had been investigated because they ha\$ indications such as systolic murmurs of doubtful significance or unexplained dyspnoea. In no case waฐ any abnormality found after an investigation which included cardiac catheterization. In these patients th mean pulmonary arterial pressure at rest ranged from 12 to $24 \mathrm{~mm}$. $\mathrm{Hg}$ (mean $18.0 \mathrm{~mm}$. $\mathrm{Hg}$ ).

The remaining 21 subjects had no history of cardio respiratory disease, and physical examination, cheș 
radiography, and pulmonary function tests showed no abnormality. The pulmonary arterial pressure was not measured in these subjects but was assumed to be normal.

GROUP B This comprised 42 patients with chronic bronchitis (38 men and 4 women), with ages ranging from 34 to 74 years. The diagnosis was based on a history of long-standing cough and sputum, for which no other cause was found. The mean pulmonary arterial pressure, which had been measured in all these patients, ranged from 18 to $74 \mathrm{~mm}$. $\mathrm{Hg}$ (mean $34.7 \mathrm{~mm}$. $\mathrm{Hg}$ ).

GROUP C This consisted of 16 patients with polycythaemia rubra vera (15 men and 1 woman) with ages ranging from 44 to 64 years. These patients have been described previously (Segel and Bishop, 1967) and both the pulmonary arterial pressure and total blood volume had been measured, in seven instances both before and after treatment with ${ }^{32} \mathrm{P}$. The mean pulmonary arterial pressure before treatment ranged from 17 to $34 \mathrm{~mm}$. $\mathrm{Hg}$ (mean $22 \mathrm{~mm}$. $\mathrm{Hg}$ ).

In all the patients in each group a technically satisfactory six-foot postero-anterior chest radiograph had been taken in the erect posture and in full inspiration within 10 days of cardiac catheterization or pulmonary function studies. Wherever possible the full inspiration position was checked with spirometer recordings of respiration at the time of exposure of the radiograph.

The transpulmonary artery distance (Fig. 1) was defined as the distance between the bifurcation of the right and left main pulmonary arteries. Simon

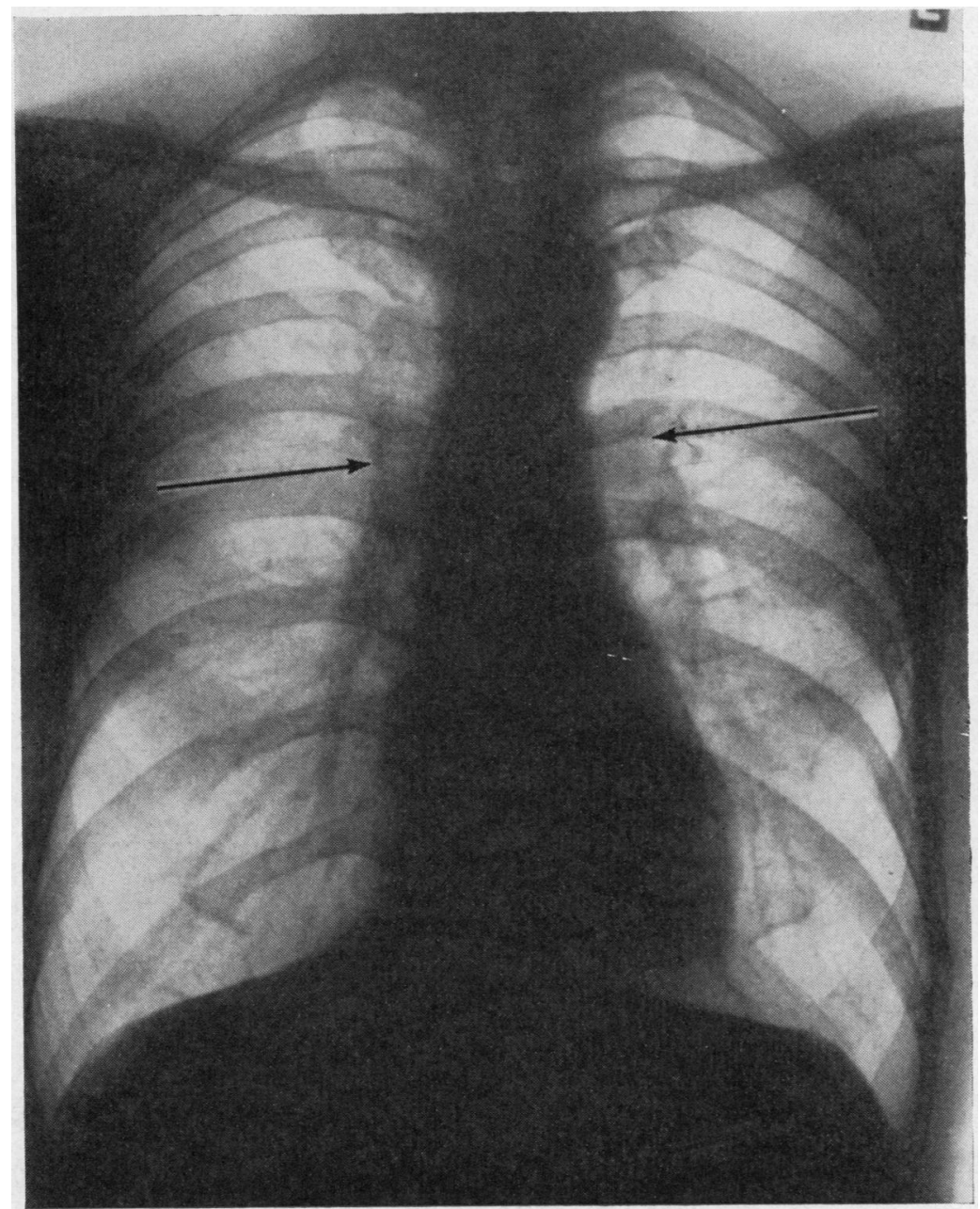

FIG. 1. Measurement of the transpulmonary artery distance on the chest radiograph between the tips of the arrows. 
(1964) used the term 'transhilar distance' to describe a similar measurement on a tomogram. Measurements of transpulmonary artery distance on the chest radiographs were made by one observer (P. H.) on three occasions separated by several weeks, and also by another observer (I. D. G.) on one occasion. All measurements were made without prior knowledge of the haemodynamic data or the results of the other observations. In one instance we were able to confirm our opinion about the anatomy of the main pulmonary arteries by comparing the plain films with those of a pulmonary angiogram. In all the patients the standing height was measured and a note was made of age and sex so that the effect of these factors on the transpulmonary artery distance could be estimated.

\section{RESULTS}

REPEATABILITY OF MEASUREMENTS Measurements were repeated on three occasions by one observer in both normal subjects and patients with chronic bronchitis. No significant difference was found between these series of measurements in five of the six comparisons made by observer $\mathbf{A}$ (see Table).

\section{T A B L E}

MEAN VALUES OF TRANSPULMONARY ARTERY DISTANCE IN NORMAL PATIENTS AND IN PATIENTS WITH CHRONIC BRONCHITIS

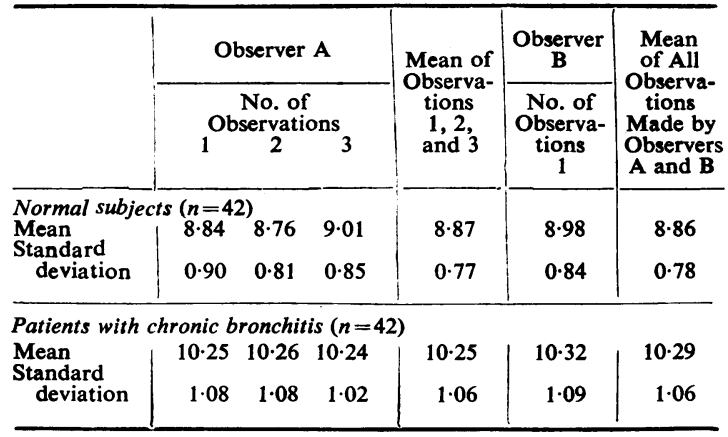

A significant difference was found between the second and third measurements in normal subjects, but it amounted to $0.16 \mathrm{~cm}$. or $1.8 \%$ of the mean measurement. The mean of the three measurements by the first observer was then compared with the single series of measurements made by the second observer and a significant difference was found. The difference was $0.1 \mathrm{~cm}$., amounting to only $1 \cdot 1 \%$ of the mean transpulmonary artery distance. In these comparisons the $t$ test was applied to individual differences between pairs of measurements, the null hypothesis being that the mean of all the differences shall be zero.
From these data the $95 \%$ confidence limits for an estimate of transpulmonary artery distance 0 representing the mean of the four measurements $\frac{\partial}{\square}$ made by two observers was $\pm 0.56 \mathrm{~cm}$. in normal $\frac{\bar{N}}{p}$ subjects and $\pm 0.36 \mathrm{~cm}$. in patients with chronic $\Phi$ bronchitis. Comparable $95 \%$ confidence limits when only two measurements by one observer (P. H.) were made were \pm 0.40 and $\pm 0.12 \mathrm{~cm}$. respectively.

GROUP A: NORMAL SUBJECTS In 42 normal $\overrightarrow{\vec{x}}$ subjects (Fig. 2) the range of the transpulmonary artery distance was $7.0-10.5 \mathrm{~cm}$., mean $8.76 \mathrm{~cm}$., S.D. $0.81 \mathrm{~cm}$. There was no correlation between the transpulmonary artery distance and age, and $\vec{\circ}$ no difference between the mean value in men and o

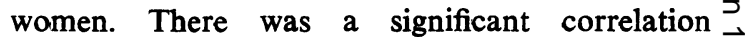
between the transpulmonary artery distance and $c$ height $(\mathrm{r}=0.54 ; \quad \mathrm{P}<0.001)$ which could be expressed by the regression equation: Transpul- $\vec{\bullet}$ monary artery distance $(\mathrm{cm})=.0.035 \times$ Height $(\mathrm{cm}$.) $+2 \cdot 8$.

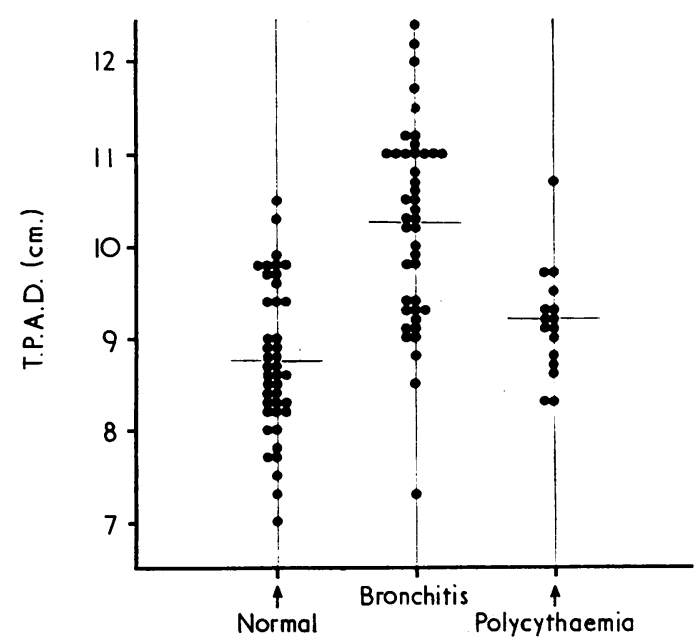

FIG. 2. Values of transpulmonary artery distance (T.P.A.D.) in cm., obtained by one observer on one occasion in 42 normal subjects, 42 patients with chronic $N$ bronchitis, and 16 patients with polycythaemia rubra vera. Mean values for each group are represented by a horizontal line in each column.

GROUP B: PATIENTS WITH CHRONIC BRONCHITIS In the 42 patients with chronic bronchitis (Fig. 2) the range of the transpulmonary artery distance was $\overrightarrow{\mathbb{D}}$ $7 \cdot 3-12.4 \mathrm{~cm}$., mean $10.26 \mathrm{~cm}$., S.D. $1.08 \mathrm{~cm}$. The $\frac{\rho}{\Phi}$ mean value was significantly greater than the mean $\varrho$ value in normal subjects $(t=6.83, \quad P<0.001)$. Individual values for transpulmonary artery $\rho$ 


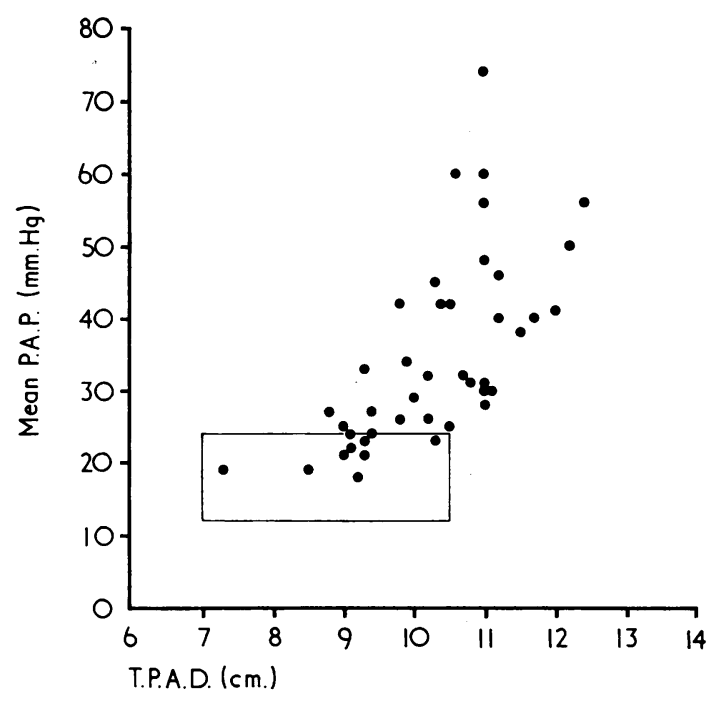

FIG. 3. Relation between transpulmonary artery distance (T.P.A.D.) and mean pulmonary arterial pressure (P.A.P.) in 42 patients with chronic bronchitis. Values for transpulmonary artery distance are those obtained by one observer in a single observation in each case. Normal ranges of transpulmonary artery distance and mean pulmonary arterial pressure are also shown.

distance are plotted against mean pulmonary arterial pressure at rest in Figure 3. A linear relationship was found between the two measurements $(r=0.66 ; P<0.001)$, the regression equation being: Mean pulmonary arterial pressure $(\mathrm{mm}$. $\mathrm{Hg})=8.0 \times$ Transpulmonary artery distance $(\mathrm{cm}$.) $-47 \cdot 0$. No improvement resulted from attempts to fit a curved line to the data. When the values for transpulmonary artery distance were adjusted for height according to the relationship derived in normal subjects, there was no significant improvement in the precision with which pulmonary arterial pressure could be predicted.

In 32 of these patients measurements of lung volumes were also available. No correlation was found between the transpulmonary artery distance and either total lung capacity, residual volume, or the ratio of residual volume to total lung capacity.

GROUP C: PATIENTS WITH POLYCYTHAEMIA RUBRA VERA In the 16 patients with polycythaemia rubra vera the range of transpulmonary artery distance was $8.3-10.7 \mathrm{~cm}$., mean $9.20 \mathrm{~cm}$., S.D. $0.57 \mathrm{~cm}$., which was not significantly different from the normal value $(t=1 \cdot 40, P>0 \cdot 1)$. In seven patients transpulmonary artery distance and mean pulmonary arterial pressure were measured both before and after treatment. There was no significant change in transpulmonary artery distance (mean before treatment $9.35 \mathrm{~cm}$.; mean after treatment $9.25 \mathrm{~cm}$.) or mean arterial pressure (mean before treatment $22.9 \mathrm{~mm}$. $\mathrm{Hg}$; mean after treatment $20.9 \mathrm{~mm} . \mathrm{Hg}$ ). In five of these patients central blood volume was measured before and after treatment and this fell from a mean of $820 \mathrm{ml} . / \mathrm{m}^{2}{ }^{2}$ to $590 \mathrm{ml} . / \mathrm{m}^{2}{ }^{2}$ In our five patients the transpulmonary artery distance was not affected by variations in central blood volume within these limits.

\section{DISCUSSION}

In this study we have shown that the transpulmonary artery distance may be measured on chest radiographs with good repeatability, and with only a small inter-observer error. The precision of the measurement was greater in the patients with chronic bronchitis than in the normal subjects. This is probably due to the clearer definition of the pulmonary arteries in the bronchitic patients. This was particularly so when emphysema, as judged by changes in the peripheral lung vessels (Laws and Heard, 1962) and bullous air-spaces, was clearly observed. There was no relation between the transpulmonary artery distance and the age or sex of the normal subjects, but a significant relation was found with the height of the same subjects.

The transpulmonary artery distance was significantly greater in the group of patients with chronic bronchitis than in the normal subjects. Individual values in these patients showed a relationship to mean pulmonary arterial pressure which appeared curvilinear, although the data could be fitted adequately to a straight line. Despite the association between height and transpulmonary artery distance which existed in the normal subjects, the incorporation of this did not make the relationship between the transpulmonary artery distance and pulmonary arterial pressure in the patients with bronchitis any closer.

It is evident that the linear relationship which has been derived can only be used to forecast the mean pulmonary artery pressure from measured transpulmonary artery distance within fairly wide limits. Inspection of Fig. 3 shows, however, that of the 13 patients with a transpulmonary artery distance of $9.5 \mathrm{~cm}$. or less, only one had a mean pulmonary arterial pressure above $30 \mathrm{~mm}$. $\mathrm{Hg}$. In the 29 patients whose transpulmonary artery distance was greater than $9.5 \mathrm{~cm}$. the mean pulmonary arterial pressure was greater than 30 
$\mathrm{mm} . \mathrm{Hg}$ in all but eight instances. While measurements of transpulmonary artery distance will not permit accurate prediction of mean pulmonary arterial pressure, it is possible to identify those patients with pulmonary hypertension with reasonable certainty. The reliability of the measurement probably compares favourably with other radiological signs in patients with chronic bronchitis and emphysema, and may well be of value in epidemiological or other studies concerned with groups of patients.

The central blood volume of a small number of patients with polycythaemia rubra vera was reduced by treatment with ${ }^{32} \mathbf{P}$ but the transpulmonary artery distances and the mean pulmonary arterial pressures were not significantly altered. This finding suggested that central blood volume is not a significant factor in determining the size of the transpulmonary artery distance, in which case the most important factor determining the size of the main pulmonary arteries is the pressure within them.

Milne (1963) has also reported a linear relation between the size of the main pulmonary artery, as determined from the radiograph by another method, and mean pulmonary arterial pressure in patients with mitral stenosis. He observed that the main pulmonary arteries appear to dilate in proportion to an increasing pulmonary artery pressure up to a level of $45-50 \mathrm{~mm}$. $\mathrm{Hg}$, but that above this level the same increment of pressure produced progressively smaller degrees of dilatation of the $\overrightarrow{\bar{z}}$ main pulmonary artery. To some extent this seemso to be the case in the patients with chronicbronchitis in this study, and it appears that at a⿳亠丷厂 level of about $45-50 \mathrm{~mm}$. $\mathrm{Hg}$ the pulmonary arteryळ is beginning to approach the limits of its distensibility.

We gratefully acknowledge a grant from the Endowment Research Fund of the United Birminghamw Hospitals, which supported this work.

\section{REFERENCES}

Abraham, A. S., Hedworth-Whitty, R. B., and Bishop, J. M. (1967) Effects of acute hypoxia and hypervolaemia singly and together upon the pulmonary circulation in patients with chronic bronchitis. Clin. Sci., 33, 371 .

Bishop, J. M., Harris, P., and Segel, N. (1965). The circulatory effects of bradykinin in normal subjects and patients with chronicbronchitis. Brit. J. Pharmacol., 25, 456.

Laws, J. W., and Heard, B. E. (1962). Emphysema and the ches film: a retrospective radiological and pathological study. Brit. J. Radiol., 35, 750.

Millard, F. J. C. (1967). The electrocardiogram in chronic lungoึ disease. Brit. Heart J., 29, 43.

Milne, E. N. C. (1963). Physiological interpretation of the plain radiograph in mitral stenosis, including a review of criteria for the radiological estimation of pulmonary arterial and venouse pressures. Brit. J. Radiol., 36, 902.

Segel, N., and Bishop, J. M. (1965). The circulatory effects of pro nethalol with special reference to changes in heart rate and stroken volume during exercise. Clin. Sci., 29, 363.

- (1966). The circulation in patients with chronic bron chitis and emphysema at rest and during exercise, with speciat reference to the influence of changes in blood viscosity and blood volume on the pulmonary circulation. J. clin. Invest., 45, 1555. rest and during Circulatory studies in polycyt

Simon, G. (1964). Radiology and emphysema. Clin. Radiol., 15, 293 